\title{
On Compact Complex Manifolds with Finite Automorphism Group
}

by

\author{
Konrad CZAJA
}

Presented by Józef SICIAK

\begin{abstract}
Summary. It is known that compact complex manifolds of general type and Kobayashi hyperbolic manifolds have finite automorphism groups. We give criteria for finiteness of the automorphism group of a compact complex manifold which allow us to produce large classes of compact complex manifolds with finite automorphism group but which are neither of general type nor Kobayashi hyperbolic.
\end{abstract}

Notations and conventions. Our notation and terminology are standard. We assume that complex spaces (in the sense of Serre) are connected and have a countable base of topology. By a complex variety we mean an irreducible complex space, and a complex manifold is an irreducible, nonsingular complex space. We write $\operatorname{Hol}(X, Y)$ for the totality of holomorphic maps of $X$ into $Y$ with the compact-open topology, and $\operatorname{Aut}(X)$ is the topological group of all holomorphic automorphisms of the complex space $X$.

We say that a compact complex variety $X$ is restricted if $X$ admits a holomorphic embedding into a complex projective space $\mathbb{P}_{m}$ for some $m$. By a complex continuum we mean a connected complex space of finite dimension.

We denote by $\operatorname{kod}(X)$ the Kodaira dimension of a compact complex manifold $X$, and by $\Pi_{1}(X)$ the fundamental group of $X$.

We say that a compact complex manifold $X$ is a Galois manifold if the fundamental group $\Pi_{1}(X)$ is finite; if this group is moreover abelian, we say that $X$ is an abelian Galois manifold.

If $a(X):=\# \operatorname{Aut}(X)<\infty$ we say that $X$ is modest; if moreover $X$ is abelian Galois then $X$ is very modest. A variety $X$ is called primary if

2000 Mathematics Subject Classification: Primary 32M99.

Key words and phrases: manifolds with finite automorphism group, non-Kähler manifolds. 
it admits no primary decomposition $X=Z_{1}^{r_{1}} \times \cdots \times Z_{q}^{r_{q}}$, where $q \geq 2$, $r_{1}, \ldots, r_{q}$ are positive integers.

Results. We start by recalling Theorem 3 of [2], stating that if $X$ and $Y$ are compact complex spaces such that $a(X)$ and $a(Y)$ are finite, then $a(X) a(Y)$ divides $a(X \times Y)$, i.e. there exists a natural number $b(X \times Y)$ such that $a(X \times Y)=b(X \times Y) a(X) a(Y)$.

From this we can infer the following corollaries.

Corollary 1. Let $X$ and $Y$ be compact modest complex continua. Then

(1) $X \times Y$ is modest and $a(X \times Y)=b(X \times Y) a(X) a(Y)$, where $b(X \times Y)$ is a positive integer.

(2) Assume that $W_{1}^{r_{1}} \times \cdots \times W_{p}^{r_{p}}$ and $Z_{1}^{s_{1}} \times \cdots \times Z_{q}^{s_{q}}$ are primary decompositions of $X$ and $Y$ respectively such that $W_{k}$ is not biholomorphic to $W_{l}$ for $k \neq l$ and $Z_{i}$ is not biholomorphic to $Z_{j}$ for $i \neq j$. If $\left\{W_{1}, \ldots, W_{p}\right\} \cap\left\{Z_{1}, \ldots, Z_{q}\right\}=\emptyset$ then $b(X \times Y)=1$; in this case we say that $X$ and $Y$ are relatively prime.

If $\left\{W_{1}, \ldots, W_{p}\right\} \cap\left\{Z_{1}, \ldots, Z_{q}\right\}=\left\{T_{1}, \ldots, T_{m}\right\}$, then

$$
b(X \times Y)=\prod_{k=1}^{m}\left(\begin{array}{c}
r_{k}+s_{k} \\
r_{k}
\end{array}\right) .
$$

Corollary 2. Let $X$ and $Y$ be relatively prime compact modest varieties. Then $a(X \times Y)=a(X) a(Y)$.

COROLlary 3. Let $X$ be a compact modest variety and let $n$ be an arbitrary positive integer. Then $a\left(X^{n}\right)=n ! a(X)^{n}$.

From the above and from the well known result of Hurwitz we infer that if $X$ is a compact complex curve of genus $g \geq 2$ then for any positive integer $n$ we have the estimate $a\left(X^{n}\right) \leq n ![84(\operatorname{gen}(X)-1)]^{n}$, and equality holds if $X$ is the Klein curve given by the equation $x_{0}^{3} x_{1}+x_{1}^{3} x_{2}+x_{1}^{3} x_{0}=0$.

COROllary 4. Let $X$ be a primary compact variety such that $a(X)=1$. Then for any positive integer $n$ we have $a\left(X^{n}\right)=n$ !, and $\operatorname{Aut}\left(X^{n}\right)$ is isomorphic to the permutation group on $n$ symbols.

Proposition 5. Let $n$ be an integer such that $n \geq 3$ and $n+2$ is a prime. Suppose that $\alpha$ is a primitive root of 1 of order $n+2$.

(1) The mapping

$\mu_{\alpha}: Z_{n+2} \times \mathbb{P}_{n} \ni(k,[x]) \mapsto\left[\alpha^{k} \cdot x_{1}, \ldots, \alpha^{k s} \cdot x_{s}, \ldots, \alpha^{k(n+1)} \cdot x_{n+1}\right] \in \mathbb{P}_{n}$ is a holomorphic action of the additive group of nonnegative integers modulo $n+2$. 
(2) The Fermat hypersurface $\mathbb{E}:=\left\{[x] \in \mathbb{P}_{n}: x_{1}^{n+2}+\cdots+x_{n+1}^{n+2}=0\right\}$ is nonsingular and simply connected, and the restriction of $\mu_{\alpha}$ to $Z_{n+2} \times \mathbb{E}$ is a well defined free holomorphic action of $Z_{n+2}$ on $\mathbb{E}$.

(3) The canonical projection $p: \mathbb{E} \rightarrow B:=\mathbb{E} / Z_{n+2}$ is a holomorphic universal covering of the restricted manifold $B$. Hence $\Pi_{1}(B) \simeq Z_{n+2}$.

(4) Any Godeaux manifold (i.e. a complex manifold biholomorphic to B) is very modest.

Proof. From [8] we infer $\operatorname{kod}(\mathbb{E})=\operatorname{kod}(B)$. We have $\operatorname{deg} \mathbb{E}-(n+1)>0$, hence $\operatorname{kod}(\mathbb{E})=\operatorname{dim}(\mathbb{E})$ and so $Y$ is modest.

The class of very modest manifolds is large as shown by

Proposition 6. Suppose that $Y$ is a Godeaux manifold and let $m$ be a positive integer. Then there exists a very modest restricted manifold $X$ such that

(1) $\operatorname{kod}(X)=\operatorname{dim} X$,

(2) $b_{2}(X) \geq b_{2}(Y)+m$, where $b_{2}(X)$ denotes the second Betti number.

Proof. Let $f: X \rightarrow Y$ be a holomorphic surjection such that $X$ is a complex manifold and there exists a point $y \in Y$ such that $f^{-1}(y)$ is biholomorphic to $\mathbb{P}_{n-1}$ and $f \mid X \backslash f^{-1}(y): X \backslash f^{-1}(y) \rightarrow Y \backslash\{y\}$ is biholomorphic. We call such a map a dilatation of $Y$. It is well known that $X$ is a restricted manifold and from [6, Satz 1.15] we infer that $b_{2}(X) \geq b_{2}(Y)+1$. By a succession of $m$ dilatations we obtain a holomorphic modification $f: X \rightarrow Y$ such that $b_{2}(X) \geq b_{2}(Y)+m$.

Observe that $\operatorname{kod}(X)=\operatorname{kod}(Y)$. From [3] we know that $\Pi_{1}(X)=\Pi_{1}(Y)$.

Now we prove that there exists a large class of modest manifolds for which $\operatorname{kod}(X)<\operatorname{dim} X$.

Proposition 7. Let $n$ be a positive integer. Then for any positive integer $k$ such that $n=2 q+k$ for some positive integer $q$, there exists a restricted manifold $Y$ for which the following conditions are satisfied:

(1) $\operatorname{dim} Y=n$,

(2) $\operatorname{kod}(Y)=k$,

(3) $Y$ is modest.

Proof. Let $B$ be a restricted modest surface such that $\operatorname{kod}(B)=0$ (examples of such surfaces are given in [4] and [5]). Let $F$ be a restricted manifold of dimension $k$ such that $\operatorname{kod}(F)=k$. If we put $Y:=B^{q} \times F$ then $\operatorname{kod}(Y)=q \cdot \operatorname{kod}(B)+\operatorname{kod}(F)=k$. From Corollary 1 we conclude that $Y$ is modest.

Modest manifolds constructed above are restricted, hence Kähler. Below we show that modest Kähler manifolds form a small subclass of modest manifolds. 
Proposition 8. Let $n$ be an integer $\geq 3$ and let $k$ be a positive integer such that $n=2 q+k$ for some positive integer $q$. Then there exists a compact complex manifold $Y$ for which the following conditions are satisfied:

(1) $\operatorname{dim} Y=n$,

(2) $\operatorname{kod}(Y)=k$,

(3) $Y$ is modest,

(4) $Y$ is complex complete algebraic,

(5) $Y$ is non-Kähler.

Proof. Let $B$ be a modest restricted surface such that $\operatorname{kod}(B)=0$. Let $M$ be a restricted 3 -dimensional manifold. Then by [7, Chapter VI, $\S 4$, Exercise 6], there exists a complex complete algebraic manifold $N$ which is bimeromorphic to $M$ and moreover $N$ is not restricted. Let $F:=N \times S$, where $S$ is a restricted manifold such that $\operatorname{kod}(S)=\operatorname{dim} S=k$, and put $Y:=$ $B^{q} \times F$. If $q$ is such that $n=2 q+k$ then condition (1) holds. Hence $\operatorname{kod}(Y)=$ $q \cdot \operatorname{kod}(B)+\operatorname{kod}(F)=k \cdot$ Applying Corollary 1 to the equality $Y=B^{q} \times F$ we infer that $Y$ is modest. Now observe that $B$ and $F$ are complete algebraic, hence $Y$ is complete algebraic. The fact that $F$ is complete algebraic implies that $F$ is Moishezon; but a Moishezon manifold admits a Kähler structure iff it is restricted. Hence we infer that $F$ is non-Kähler.

A product of compact complex manifolds is Kähler iff each factor is Kähler, hence we conclude that $Y$ is non-Kähler.

Now by applying Corollary 1 we get

Corollary 9. Let $X$ be a compact modest manifold and let $Y$ be as in Proposition 8. Then $X \times Y$ is a modest non-Kähler manifold.

Corollary 9 shows that compact modest Kähler manifolds form a very small subclass of compact modest manifolds.

If $f: X \rightarrow S$ is a smooth holomorphic surjective map such that $S$ and all fibres are compact with discrete automorphism group, then $\operatorname{Aut}(X)$ is discrete. The proof is in principle the same as in the locally trivial case in [1]. Hence the following problem arises:

Given a smooth holomorphic surjective map $f: X \rightarrow S$ such that $S$ and all fibres are compact modest, is $X$ also modest?

\section{References}

[1] S. Cynk and T. Szemberg, On Urata fibre bundles, Bull. Polish Acad. Sci. Math. 45 (1997), 297-301.

[2] K. Czaja, Two results on automorphism groups of complex manifolds, ibid. 39 (1991), 133-135.

[3] Ph. Griffiths and J. Harris, Principles of Algebraic Geometry, Dekker, 1978. 
[4] S. Kondo, Enriques surfaces with finite automorphism groups, Japan J. Math. 12 (1986), 191-282.

[5] - , Algebraic K3 surfaces with finite automorphism groups, Nagoya Math. J. 116 (1989), 1-15.

[6] R. Remmert und T. Van de Ven, Über holomorphe Abbildungen projektiv-algebraischer Mannigfaltigkeiten auf komplexe Räume, Math. Ann. 142 (1961), 453-486.

[7] I. R. Shafarevich, Basic Algebraic Geometry, Springer, 1977.

[8] K. Ueno, Classification Theory of Algebraic Varieties and Compact Complex Spaces, Lecture Notes in Math. 439, Springer, 1975.

Konrad Czaja

Faculty of Mathematics and Natural Sciences

Cardinal Stefan Wyszyński University

Dewajtis 5

01-815 Warszawa, Poland

Received June 7, 2004;

received in final form January 31, 2005 